\title{
From geometric optimization and nonsmooth analysis to distributed coordination algorithms
}

\author{
Jorge Cortés Francesco Bullo \\ Coordinated Science Laboratory \\ University of Illinois at Urbana-Champaign \\ Urbana, IL 61801, United States \\ \{jcortes, bullo\}@uiuc.edu, http://motion.csl.uiuc.edu
}

\begin{abstract}
We investigate the coordination of groups of autonomous robots performing spatially-distributed sensing tasks. We present facility location functions from geometric optimization and study their differentiable properties. We then design distributed coordination algorithms and analyze them as nonsmooth gradient flows. The resulting control laws correspond to basic interaction behaviors between the robots. The technical approach relies on concepts from computational geometry, nonsmooth analysis, and the dynamical system approach to algorithms.
\end{abstract}

\section{INTRODUCTION}

The deployment of large groups of autonomous vehicles is rapidly becoming possible because of technological advances in computing, networking, and miniaturization. Future multivehicle networks will coordinate their actions to perform challenging spatially-distributed tasks (e.g., search and recovery, exploration, and environmental monitoring for pollution detection and estimation). This scenario motivates the study of algorithms for autonomy, adaptation, and coordination of multi-vehicle networks. Our approach is to design networkwide performance measures which encode meaningful sensing tasks. At the same time, we consider all constraints on the network behavior. Coordination algorithms need to be adaptive and distributed in order for the resulting network to be scalable, to tolerate failures, and to adapt to changing environments, topologies and tasks.

In this paper, we consider a facility location function encoding a coverage performance criteria. A multi-vehicle network provides optimal coverage of a domain of interest if it minimizes the largest distance from any point in the domain to one of the vehicle locations. In other words, if $\left(p_{1}, \ldots, p_{n}\right)$ are the vehicles locations in a domain $Q$, we minimize the multi-center function

$$
\max _{q \in Q}\left[\min _{i \in\{1, \ldots, n\}}\left\|q-p_{i}\right\|^{2}\right] .
$$

We study its differentiable properties via nonsmooth analysis [1]. We show it is locally Lipschitz and regular, and compute its generalized gradient. Under a technical condition, we show that the local minima of the multi-center function are center Voronoi configurations. Next, we design distributed algorithms following a dynamical systems approach; see [2], [3]. We study the corresponding nonsmooth gradient flow using the tools in [4], [5], [6]. Although this flow has some convergence properties, it is not amenable to a distributed implementation. Drawing connections with quantization theory [7], [8], [9], we consider two distributed coordination algorithms: a novel strategy based on the generalized gradient and a strategy similar to the well-known Lloyd algorithm. Both coordination algorithms are guaranteed to continuously improve the network performance. We investigate their asymptotic behavior. In the journal version of this work [10], we provide the proofs for all statements in this note. Two of our results are related to well-known conjectures in the locational optimization literature [11], [12]: (i) that the multicenter problem is equivalent to a disk covering problem, and (ii) that the generalized Lloyd strategy converges to the set of center Voronoi configurations.

The paper is organized as follows. Section II establishes the key concepts on Voronoi partitions, multi-center problems, nonsmooth and stability analysis. Section III presents a complete treatment for the 1-center problem. Section IV discusses the differentiable properties and the critical points of the multi-center function. Section $\mathrm{V}$ introduces various coordination algorithms (distributed and non-distributed) and discusses their correctness. We gather our conclusions in Section VI.

\section{PRELIMINARIES AND PROBLEM SETUP}

Let $\|\cdot\|$ denote the Euclidean distance function on $\mathbb{R}^{N}$ and let $v \cdot w$ denote the scalar product of $v, w \in \mathbb{R}^{N}$. Let $\operatorname{versus}(v)$ denote the unit vector in the direction of $0 \neq v \in \mathbb{R}^{N}$, i.e., $\operatorname{versus}(v)=v /\|v\|$. Given $S \subset \mathbb{R}^{N}$, $\operatorname{co}(S)$ and int $S$ denote its convex hull and interior set, respectively. If $S$ is convex, let $\operatorname{proj}_{S}: \mathbb{R}^{N} \rightarrow S$ denote the orthogonal projection onto $S$ and let $\mathrm{D}_{S}: \mathbb{R}^{N} \rightarrow \mathbb{R}$ denote the distance function to $S$. For $R>0, \bar{B}_{N}(p, R)=$ $\left\{q \in \mathbb{R}^{N} \mid\|p-q\| \leq R\right\}$ and $B_{N}(p, R)=\operatorname{int} \bar{B}_{N}(p, R)$. Let $Q$ be a convex polygon in $\mathbb{R}^{2}$. We denote by $\operatorname{Ed}(Q)=$ $\left\{e_{1}, \ldots, e_{M}\right\}$ and $\operatorname{Ve}(Q)=\left\{v_{1}, \ldots, v_{L}\right\}$ the set of edges and vertices of $Q$, respectively. Let $P=\left(p_{1}, \ldots, p_{n}\right) \in$ $Q^{n} \subset\left(\mathbb{R}^{2}\right)^{n}$ denote the location of $n$ generators in $Q$. Let $\pi_{i}: Q^{n} \rightarrow Q$ be the canonical projection onto the $i$ th factor. 


\section{A. Voronoi partitions}

We refer the reader to [13], [14] for comprehensive treatments on Voronoi diagrams. The Voronoi partition $\mathcal{V}(P)=\left(V_{1}(P), \ldots, V_{n}(P)\right)$ of $Q$ generated by the points $\left(p_{1}, \ldots, p_{n}\right)$ is defined by

$$
V_{i}(P)=\left\{q \in Q \mid\left\|q-p_{i}\right\| \leq\left\|q-p_{j}\right\|, \forall j \neq i\right\} .
$$

For simplicity, we refer to $V_{i}(P)$ as $V_{i}$. Since $Q$ is a convex polygon, the boundary of each $V_{i}$ is the union of a finite number of segments. If $V_{i}$ and $V_{j}$ share an edge, then $p_{i}$ is a (Voronoi) neighbor of $p_{j}$ (and vice-versa). All Voronoi neighboring relations are encoded in the mapping $\mathcal{N}: Q^{n} \times$ $\{1, \ldots, n\} \rightarrow 2^{\{1, \ldots, n\}}$ where $\mathcal{N}(P, i)$ is the set of indexes of the Voronoi neighbors of $p_{i}$. We will often omit $P$ and instead write $\mathcal{N}(i)$.

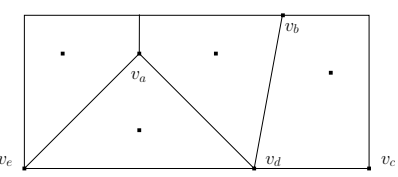

Fig. 1. A Voronoi partition with degenerate and nondegenerate vertices. Vertices $v_{a}, v_{b}$, and $v_{c}$ are nondegenerate vertices of type (a), (b), (c), respectively. Vertices $v_{d}$ and $v_{e}$ are degenerate.

For $P \in Q^{n}$, the vertices $v$ of $\mathcal{V}(P)$ are classified as follows: $v$ is of type $(a)$ if it is the center of the circle passing through three generators (say, $p_{i}, p_{j}$, and $p_{k}$ ), $v$ is of type ( $b$ ) if it is the intersection between an edge of $Q$ and the bisector determined by two generators (say, $e, p_{i}$, and $p_{j}$ ), and $v$ is of type (c) if it is a vertex of $Q$, i.e., it is determined by two edges of $Q$ and the generator of a cell containing it (say, $e, f$, and $\left.p_{i}\right)$. Correspondingly, we write $v(i, j, k), v(e, i, j)$, and $v(e, f, i)$. The vertex $v \in \operatorname{Ve}\left(V_{i}(P)\right)$ is nondegenerate if it is determined by exactly three elements, otherwise it is degenerate. The configuration $P$ is nondegenerate at the ith generator if all vertices $v \in \operatorname{Ve}\left(V_{i}(P)\right)$ are nondegenerate, otherwise $P$ is degenerate at the ith generator. Finally, $P$ is nondegenerate if all its vertices are nondegenerate, otherwise it is degenerate (see Fig. 1).

\section{B. Disk-covering and sphere-packing problems}

We are interested in the facility location problems

$$
\begin{aligned}
& \min _{p_{1}, \ldots, p_{n}}\left\{\max _{q \in Q}\left\{\min _{i \in\{1, \ldots, n\}}\left\|q-p_{i}\right\|\right\}\right\}, \\
& \max _{p_{1}, \ldots, p_{n}}\left\{\min _{\substack{i, j \in\{1, \ldots, n\} \\
i \neq j, e \in \operatorname{Ed}(Q)}}\left\{\frac{1}{2}\left\|p_{i}-p_{j}\right\|, \mathrm{D}_{e}\left(p_{i}\right)\right\}\right\} .
\end{aligned}
$$

The problem (1) is referred to as the $p$-center problem in [12], [11]. Along paper, we refer to it as the multicenter problem. In the context of coverage control for mobile networks [15], it corresponds to the worst case scenario, in which no information is available on the events taking place in $Q$. The network then tries to minimize the largest possible distance of any point in $Q$ to one of the generators' locations, i.e. to minimize,

$$
\begin{aligned}
\mathcal{H}_{\mathrm{DC}}(P) & =\max _{q \in Q}\left\{\min _{i \in\{1, \ldots, n\}}\left\|q-p_{i}\right\|\right\} \\
& =\max _{i \in\{1, \ldots, n\}}\left\{\max _{q \in V_{i}}\left\|q-p_{i}\right\|\right\} .
\end{aligned}
$$

It is conjectured in [11] that this problem can be restated as a disk-covering problem: how to cover a region with disks of minimum radius. In Theorem 4.3 we provide a positive answer to this question. Given a polytope $W$ in $\mathbb{R}^{N}$, its circumcenter, $\mathrm{CC}(W)$, is the center of the minimum-radius sphere that contains $W$. We say that $P$ is a circumcenter Voronoi configuration if $p_{i}=\mathrm{CC}\left(V_{i}(P)\right)$, for all $i \in$ $\{1, \ldots, n\}$.

We refer to (2) as the multi-incenter problem. It corresponds to the situation where the network tries to maximize the coverage of $Q$ so that the sensing radius do not overlap. We then consider the maximization of

$$
\mathcal{H}_{\mathrm{SP}}(P)=\min _{\substack{i, j \in\{1, \ldots, n\} \\ i \neq j, e \in \operatorname{Ed}(Q)}}\left\{\frac{1}{2}\left\|p_{i}-p_{j}\right\|, \mathrm{D}_{e}\left(p_{i}\right)\right\}
$$

A similar conjecture is that this is a sphere-packing problem: how to maximize the coverage of a region with nonoverlapping disks of minimum radius. Here we confine ourselves to the multicenter problem.

\section{Nonsmooth analysis}

Here we review some facts on nonsmooth analysis [1]. The right directional derivative of $f$ at $x$ in the direction of $v \in \mathbb{R}^{N}$ is

$$
f^{\prime}(x, v)=\lim _{t \rightarrow 0^{+}} \frac{f(x+t v)-f(x)}{t},
$$

when this limits exists. The generalized directional derivative of $f$ at $x$ in the direction of $v \in \mathbb{R}^{N}$ is

$$
f^{o}(x ; v)=\limsup _{\substack{y \rightarrow x \\ t \rightarrow 0^{+}}} \frac{f(y+t v)-f(y)}{t} .
$$

Definition 2.1: $f: \mathbb{R}^{N} \rightarrow \mathbb{R}$ is regular at $x \in \mathbb{R}^{N}$ if for all $v \in \mathbb{R}^{N}, f^{\prime}(x ; v)$ exists and $f^{o}(x ; v)=f^{\prime}(x ; v)$.

From Rademacher's Theorem [1], locally Lipschitz functions are differentiable almost everywhere. If $\Omega_{f}$ denotes the set of points in $\mathbb{R}^{N}$ where $f$ fails to be differentiable, the generalized gradient of $f$ is

$$
\partial f(x)=\operatorname{co}\left\{\lim _{i \rightarrow+\infty} d f\left(x_{i}\right) \mid x_{i} \rightarrow x, x_{i} \notin S \cup \Omega_{f}\right\},
$$

where $\mu(S)=0$ ( $\mu$ denotes the usual Lebesgue measure). A point $x \in \mathbb{R}^{N}$ with $0 \in \partial f(x)$ is a critical point of $f$.

Proposition 2.2: Let $\quad\left\{f_{k}: \mathbb{R}^{N} \rightarrow \mathbb{R} \mid k \in\{1, \ldots, m\}\right\}$ be a collection of locally Lipschitz functions at $x \in \mathbb{R}^{N}$. Let $f\left(x^{\prime}\right)=\min \left\{f_{k}\left(x^{\prime}\right) \mid k \in\{1, \ldots, m\}\right\}$. Then, $f$ is locally 
Lipschitz at $x$, and if $I\left(x^{\prime}\right)$ denotes the set of indexes $k$ for which $f_{k}\left(x^{\prime}\right)=f\left(x^{\prime}\right)$, we have

$$
\partial f(x) \subset \operatorname{co}\left\{\partial f_{i}(x) \mid i \in I(x)\right\},
$$

and if each $f_{i}$ is regular at $x$ for $i \in I(x)$, then equality holds and $f$ is regular at $x$.

Proposition 2.3: Let $f$ be a locally Lipschitz function at $x \in \mathbb{R}^{N}$. If $f$ attains a local minimum or maximum at $x$, then $0 \in \partial f(x)$, i.e., $x$ is a critical point.

Let Ln : $2^{\mathbb{R}^{N}} \rightarrow 2^{\mathbb{R}^{N}}$ be the set-valued mapping that associates to each $S \subset \mathbb{R}^{N}$ the set of its least-norm elements $\operatorname{Ln}(S)$. If the set $S$ is convex, then $\operatorname{Ln}(S)=\operatorname{proj}_{S}(0)$. For a locally Lipschitz function $f$, we consider the generalized gradient vector field $\operatorname{Ln}(\partial f): \mathbb{R}^{N} \rightarrow \mathbb{R}^{N}$ given by $x \mapsto$ $\operatorname{Ln}(\partial f)(x)=\operatorname{Ln}(\partial f(x))$.

Theorem 2.4: Let $f$ be a locally Lipschitz function at $x$. Assume $0 \notin \partial f(x)$. Then, there exists $T>0$ such that for all $0<t<T$

$$
f(x-t \operatorname{Ln}(\partial f)(x)) \leq f(x)-\frac{t}{2}\|\operatorname{Ln}(\partial f)(x)\|^{2} .
$$

The vector $-\operatorname{Ln}(\partial f)(x)$ is called a direction of descent.

\section{Stability analysis via nonsmooth Lyapunov functions}

For differential equations with discontinuous right-hand sides, solutions are defined in terms of differential inclusions [4]. Let $F: \mathbb{R}^{N} \rightarrow 2^{\mathbb{R}^{N}}$ be a set-valued map. A solution to the differential inclusion $\dot{x} \in F(x)$ on an interval $\left[t_{0}, t_{1}\right] \subset \mathbb{R}$ is defined as an absolutely continuous function $x:\left[t_{0}, t_{1}\right] \rightarrow \mathbb{R}^{N}$ such that $\dot{x}(t) \in F(x(t))$ for almost all $t \in\left[t_{0}, t_{1}\right]$. Now, consider the equation

$$
\dot{x}(t)=X(x(t)),
$$

where $X: \mathbb{R}^{N} \rightarrow \mathbb{R}^{N}$ is measurable and essentially locally bounded. The solution of (4) has to be understood in the Filippov sense. For each $x \in \mathbb{R}^{N}$, consider

$$
K[X](x)=\bigcap_{\delta>0} \bigcap_{\mu(S)=0} \operatorname{co}\left\{X\left(B_{N}(x, \delta) \backslash S\right)\right\} .
$$

A Filippov solution of (4) on an interval $\left[t_{0}, t_{1}\right] \subset \mathbb{R}$ is defined as a solution of the differential inclusion

$$
\dot{x} \in K[X](x) .
$$

A set $M$ is weakly invariant (resp. strongly invariant) for (4) if for each $x_{0} \in M$, contains a maximal solution (resp. all maximal solutions) of (4). Given a locally Lipschitz function $f: \mathbb{R}^{N} \rightarrow \mathbb{R}$, define the set-valued Lie derivative of $f$ with respect to $X$ at $x$ as

$$
\begin{aligned}
\widetilde{\mathcal{L}}_{X} f(x)=\{a \in \mathbb{R} \mid \exists v \in K[X](x) \text { such that } \\
\qquad \cdot v=a, \forall \zeta \in \partial f(x)\} .
\end{aligned}
$$

For each $x \in \mathbb{R}^{N}, \widetilde{\mathcal{L}}_{X} f(x)$ is a closed and bounded interval in $\mathbb{R}$, possibly empty. The following result is a generalization of LaSalle principle for differential equations of the form (4) with nonsmooth Lyapunov functions. The formulation is taken from [5], and slightly generalizes the one presented in [6].

Theorem 2.5 (LaSalle principle): Let $f: \mathbb{R}^{N} \rightarrow \mathbb{R}$ be a locally Lipschitz and regular function. Let $x_{0} \in \mathbb{R}^{N}$ and let $f^{-1}\left(\leq f\left(x_{0}\right), x_{0}\right)$ be the connected component of $\left\{x \in \mathbb{R}^{N} \mid f(x) \leq f\left(x_{0}\right)\right\}$ containing $x_{0}$. Assume either $\max \widetilde{\mathcal{L}}_{X} f(x) \leq 0$ or $\widetilde{\mathcal{L}}_{X} f(x)=\emptyset$ for all $x \in f^{-1}(\leq$ $\left.f\left(x_{0}\right), x_{0}\right)$, and that this set is bounded. Then $f^{-1}(\leq$ $\left.f\left(x_{0}\right), x_{0}\right)$ is strongly invariant for (4). Let

$$
Z_{X, f}=\left\{x \in \mathbb{R}^{N} \mid 0 \in \widetilde{\mathcal{L}}_{X} f(x)\right\} .
$$

Then, any solution $x:\left[t_{0},+\infty\right) \rightarrow \mathbb{R}^{N}$ of (4) starting from $x_{0}$ converges to the largest weakly invariant set $M$ contained in $\bar{Z}_{X, f} \cap f^{-1}\left(\leq f\left(x_{0}\right), x_{0}\right)$.

\section{E. Nonsmooth gradient flows}

Finally, we are in a position to present the nonsmooth analogue of well-known results on gradient flows. Given a locally Lipschitz and regular function $f$, consider

$$
\dot{x}(t)=-\operatorname{Ln}(\partial f)(x(t)) .
$$

Theorem 2.4 guarantees that, unless the flow is at a critical point, $-\operatorname{Ln}(\partial f)(x)$ is a direction of descent at $x$. In general, the vector field $\operatorname{Ln}(\partial f)$ is discontinuous, and therefore its solution must be understood in the Filippov sense. Since $f$ is locally Lipschitz, $\operatorname{Ln}(\partial f)=d f$ a.e. The following result guarantees the convergence to the set of critical points of $f$.

Proposition 2.6: Let $x_{0} \in \mathbb{R}^{N}$ and $f^{-1}\left(\leq f\left(x_{0}\right), x_{0}\right)$ is bounded. Then, any solution $x:\left[t_{0},+\infty\right) \rightarrow \mathbb{R}^{N}$ of eq. (6) starting from $x_{0}$ converges asymptotically to the set of critical points of $f$ contained in $f^{-1}\left(\leq f\left(x_{0}\right), x_{0}\right)$.

\section{THE 1-CENTER PROBLEM}

In this section we consider the disk-covering problem with a single generator, i.e., $n=1$. This treatment will give us the necessary insight to tackle later the more involved multicenter problem. When $n=1$, the minimization of $\mathcal{H}_{\mathrm{DC}}$ consists of finding the center of the minimum-radius sphere enclosing the polygon $Q$. Let us therefore define the function

$$
\begin{aligned}
\lg _{Q}(p) & =\max \{\|q-p\| \mid q \in Q\} \\
& =\max \{\|v-p\| \mid v \in \operatorname{Ve}(Q)\}=\mathcal{H}_{\mathrm{DC}}(p) .
\end{aligned}
$$

Since the function $\lg _{Q}$ is the maximum of a (finite) set of convex functions in $p$, it is also a convex function [16]. Therefore, any local minimum of $\lg _{Q}$ is also global. Furthermore, one can show that the function $\lg _{Q}$ has a unique global minimum, which is the circumcenter of the polygon Q.

Proposition 3.1: The function $\lg _{Q}(p)$ is locally Lipschitz and regular, and its generalized gradient is

$$
\begin{aligned}
& \partial \lg _{Q}(p)=\operatorname{co}\{\operatorname{versus}(p-v) \mid \\
& \left.v \in \operatorname{Ve}(Q), \lg _{Q}(p)=\|p-v\|\right\}
\end{aligned}
$$


Moreover, $0 \in \partial \lg _{Q}(p) \Longleftrightarrow p=\mathrm{CC}(Q)$.

Next, let us study the generalized gradient flow arising from the 1-center function. An immediate consequence of Propositions 2.6 and 3.1 is the following result.

Proposition 3.2: The gradient flow of $\lg _{Q}, \dot{x}(t)=$ - $\operatorname{Ln}\left(\partial \lg _{Q}\right)(x(t))$ converges asymptotically to the circumcenter $\mathrm{CC}(Q)$. If $0 \in \operatorname{int} \partial \lg _{Q}(\mathrm{CC}(Q))$, then the flow reaches $\mathrm{CC}(Q)$ in finite time.

Note that if $0 \in \partial \lg _{Q}(\mathrm{CC}(Q)) \backslash \operatorname{int} \partial \lg _{Q}(\mathrm{CC}(Q))$, then generically convergence is achieved over an infinite time horizon. Fig. 2 shows an example of the implementation of the gradient descent of $\lg _{Q}$. If $\mathrm{CC}(Q)$ is first computed offline, the strategy of directly going toward it would converge in a less "erratic" way.

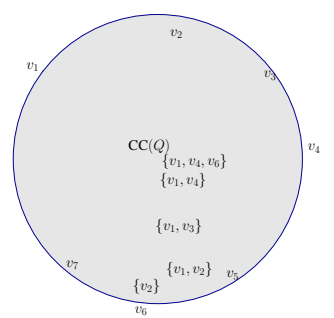

Fig. 2. Illustration of the gradient descent of $\lg _{Q}$. The points where the curve $t \mapsto p(t)$ fails to be differentiable correspond to points where there is a new vertex $v$ of $Q$ such that $\|p(t)-v\|=\lg _{Q}(p(t))$. The circumcenter is attained in finite time according to Proposition 3.2.

\section{ANALYSIS OF THE MULTI-CENTER FUNCTION}

We characterize the smoothness properties of $\mathcal{H}_{\mathrm{DC}}$ (generalized gradient, critical points) for arbitrary numbers of generators. We start by providing some alternative expressions and useful quantities. We write

$$
\mathcal{H}_{\mathrm{DC}}(P)=\max _{i \in\{1, \ldots, n\}} G_{i}(P),
$$

where $G_{i}(P)=\max _{q \in V_{i}(P)}\left\|q-p_{i}\right\|$. Note that $G_{i}(P)=$ $\lg _{V_{i}(P)}\left(p_{i}\right)$, where, for $i \in\{1, \ldots, n\}, \lg _{V_{i}}: V_{i} \rightarrow \mathbb{R}$. Proposition 3.1 provides an explicit expression for the generalized gradient of $\lg _{V_{i}}$ when $V_{i}$ is held fixed. Despite the slight abuse of notation, it is convenient to let $\partial \lg _{V_{i}(P)}\left(p_{i}\right)$ denote $\partial \lg _{V}\left(p_{i}\right)_{\mid V=V_{i}(P)}$. In contrast to this analysis at fixed Voronoi partition, the properties of $G_{i}$ are strongly affected by the dependence on $\mathcal{V}(P)$. Our objective is to characterize these properties in order to study $\mathcal{H}_{\mathrm{DC}}$.

Proposition 4.1: The functions $G_{i}: Q^{n} \rightarrow \mathbb{R}$ are locally Lipschitz and regular. As a consequence, $\mathcal{H}_{\mathrm{DC}}: Q^{n} \rightarrow \mathbb{R}$ is locally Lipschitz and regular.

We now introduce some quantities that are useful in characterizing the generalized gradient of $G_{i}$. Given a vertex of type (b), $v=v(e, i, j)$, determined by the edge $e$ and two generators $p_{i}$ and $p_{j}$, consider the scalar function $\lambda(e, i, j)$ defined by

$$
\operatorname{proj}_{e}\left(p_{j}-v(e, i, j)\right)=\lambda(e, i, j) \operatorname{proj}_{e}\left(p_{j}-p_{i}\right)
$$

where $P_{e}$ is the orthogonal projection onto the edge $e$; see Fig. 3. One can see that $\lambda(e, i, j)+\lambda(e, j, i)=1$. If $e$ is a segment in the line $a x+b y+c=0,\left(\Delta x_{i j}, \Delta y_{i j}\right)=p_{j}-p_{i}$, $\left(x_{m}, y_{m}\right)=\left(p_{i}+p_{j}\right) / 2$, then one can show

$$
\lambda(e, i, j)=\frac{1}{2}-\frac{\left(a \Delta x_{i j}+b \Delta y_{i j}\right)\left(a x_{m}+b y_{m}+c\right)}{\left(a \Delta y_{i j}-b \Delta x_{i j}\right)^{2}} .
$$

Given a vertex of type (a), $v=v(i, j, k)$, determined by
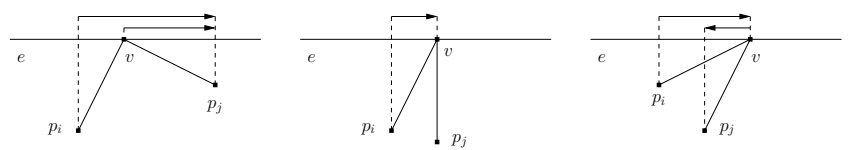

Fig. 3. To illustrate eq. (9) we draw the vectors $\operatorname{proj}_{e}\left(p_{j}-v(e, i, j)\right)$ and $\operatorname{proj}_{e}\left(p_{j}-p_{i}\right)$ for various locations of $p_{i}, p_{j}$, and $e$. The left, center and right figures correspond to $\lambda(e, i, j)>0, \lambda(e, i, j)=0, \lambda(e, i, j)<0$, resp.

the three generators $p_{i}, p_{j}$, and $p_{k}$, we consider the scalar function $\mu(i, j, k)$ defined by

$$
\operatorname{proj}_{e_{j k}}\left(p_{\ell}-v(i, j, k)\right)=\mu(i, j, k) \operatorname{proj}_{e_{j k}}\left(p_{\ell}-p_{i}\right)
$$

where $e_{j k}$ is the bisector of $p_{j}$ and $p_{k}$ and where $p_{\ell}=p_{j}$ if $p_{j}$ belongs to the half-plane defined by $e_{j k}$ containing $p_{i}$, and $p_{\ell}=p_{k}$ otherwise. One can see that $\mu(i, j, k)=$ $\mu(i, k, j)$ and that $\mu(i, j, k)+\mu(j, k, i)+\mu(k, i, j)=1$. From the expression of $\lambda$, one can obtain the next expression for $\mu(i, j, k)$,

$$
\frac{1}{2}+\frac{\left(\Delta x_{i j} \Delta x_{j k}+\Delta y_{i j} \Delta y_{j k}\right)\left(\Delta x_{i k} \Delta x_{j k}+\Delta y_{i k} \Delta y_{j k}\right)}{2\left(x_{k} \Delta y_{i j}-x_{j} \Delta y_{i k}+x_{i} \Delta y_{j k}\right)^{2}} .
$$

In general, $\lambda$ and $\mu$ are not positive functions.

Proposition 4.2: Let $P \in Q^{n}$. Then

$$
\begin{aligned}
\partial G_{i}(P) & =\operatorname{co}\left\{\partial_{v} G_{i}(P) \in\left(\mathbb{R}^{2}\right)^{n} \mid\right. \\
& \left.v \in \operatorname{Ve}\left(V_{i}(P)\right) \text { such that } G_{i}(P)=\left\|p_{i}-v\right\|\right\}
\end{aligned}
$$

where we consider separately the following cases. If $v=$ $v(i, j, k)$ is a nondegenerate vertex of type (a), then

$$
\begin{gathered}
\partial_{v(i, j, k)} G_{i}(P)=\partial_{v(k, i, j)} G_{k}(P)=\partial_{v(j, k, i)} G_{j}(P)= \\
(0, \ldots, \underbrace{\mu(i, j, k) \operatorname{versus}\left(p_{i}-v\right)}_{j \text { th place }}, \ldots, \underbrace{\mu(j, k, i) \operatorname{versus}\left(p_{j}-v\right)}_{k \text { th place }} \\
, \ldots, \underbrace{\mu(k, i, j)}_{\text {the }}, \ldots, 0)
\end{gathered}
$$

where, without loss of generality, we let $i<j<k$. If $v=$ $v(e, i, j)$ is a nondegenerate vertex of type (b), then

$$
\begin{aligned}
\partial_{v(e, i, j)} G_{i}(P) & =\partial_{v(e, j, i)} G_{j}(P) \\
=(0, \ldots, \underbrace{\lambda(e, i, j) \operatorname{versus}\left(p_{i}-v\right)}_{\text {ith place }}, \ldots, & \underbrace{\lambda(e, j, i) \operatorname{versus}\left(p_{j}-v\right)}_{j \text { th place }}, \ldots, 0)
\end{aligned}
$$




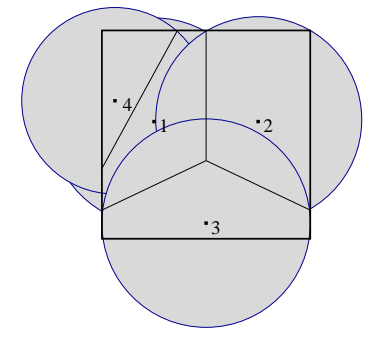

Fig. 4. Local extrema of the disk-covering function in a convex polygonal environment. The configuration corresponds to a local minimum of $\mathcal{H}_{\mathrm{DC}}$ with $0 \in \partial \mathcal{H}_{\mathrm{DC}}(P)$ and int $\partial \mathcal{H}_{\mathrm{DC}}(P)=\emptyset$. The 4 th generator is inactive and non-centered.

where, without loss of generality, we let $i<j$. If $v=$ $v(e, f, i)$ is a nondegenerate vertex of type (c), then

$$
\partial_{v(e, f, i)} G_{i}(P)=(0, \ldots, 0, \underbrace{\operatorname{versus}\left(p_{i}-v\right)}_{i \text { th place }}, 0, \ldots, 0) .
$$

Finally, if the vertex $v$ is degenerate, i.e., if $v$ is determined by $d>3$ elements (generators or edges), then there are $\left(\begin{array}{c}d-1 \\ 2\end{array}\right)$ pairs of elements which determine the vertex $v$ together with the generator $p_{i}$. In this case, $\partial_{v} G_{i}(P)$ is the convex hull of $\partial_{v(\alpha, \beta, \gamma)} G_{i}(P)$ for all $\left(\begin{array}{c}d-1 \\ 2\end{array}\right)$ such triplets $(\alpha, \beta, \gamma)$.

As a consequence of Propositions 2.2 and 4.1, we have

$$
\partial \mathcal{H}_{\mathrm{DC}}(P)=\operatorname{co}\left\{\partial G_{i}(P) \mid i \in I(P)\right\} .
$$

Theorem 4.3 (Minima of $\mathcal{H}_{D C}$ ): Let $P \in Q^{n}$ be nondegenerate and $0 \in \operatorname{int} \partial \mathcal{H}_{\mathrm{DC}}(P)$. Then, $P$ is a strict local minimum of $\mathcal{H}_{\mathrm{DC}}$, all generators are active and $P$ is a circumcenter Voronoi configuration.

Remark 4.4: Theorem 4.3 provides the interpretation of the multicenter problem that we gave in Section II-B: since all generators are active, they share the same radius. If one drops the hypothesis $0 \in \operatorname{int} \partial \mathcal{H}_{\mathrm{DC}}(P)$, one can think of simple examples where $P$ is a local minimum of $\mathcal{H}_{\mathrm{DC}}$, and there are generators which are inactive and non-centered, e.g. Fig. 4.

\section{DYNAMICAL SYSTEMS FOR THE MULTI-CENTER PROBLEM}

Here, we describe three algorithms that (locally) extremize the multi-center function for the disk-covering problem.We present continuous-time versions of the algorithms and discuss their convergence properties. The generators' location obeys a first order dynamical behavior described by

$$
\dot{p}_{i}=u_{i}\left(p_{1}, \ldots, p_{n}\right), \quad i \in\{1, \ldots, n\} .
$$

The dynamical system (11) is said to be centralized if there exists at least an $i \in\{1, \ldots, n\}$ such that $u_{i}\left(p_{1}, \ldots, p_{n}\right)$ cannot be written as a function of the form $u_{i}\left(p_{i}, p_{i_{1}}, \ldots, p_{i_{m}}\right)$, with $m<n-1$. The dynamical system (11) is said to be Voronoi-distributed if each $u_{i}\left(p_{1}, \ldots, p_{n}\right)$ can be written as a function of the form $u_{i}\left(p_{i}, p_{i_{1}}, \ldots, p_{i_{m}}\right)$, with $i_{k} \in \mathcal{N}(P, i)$, $k \in\{1, \ldots, m\}$. We refer to [15] for more details on the distributed character of Voronoi neighborhood relationships.

\section{A. Nonsmooth gradient dynamical systems}

Consider the generalized gradient descent flow (6) for the locational optimization functions $\mathcal{H}_{\mathrm{DC}}$,

$$
\dot{P}=-\operatorname{Ln}\left(\partial \mathcal{H}_{\mathrm{DC}}\right)(P) \text {. }
$$

Alternatively, we may write for each $i \in\{1, \ldots, n\}$,

$$
\dot{p}_{i}=-\pi_{i}\left(\operatorname{Ln}\left(\partial \mathcal{H}_{\mathrm{DC}}\right)\left(p_{1}, \ldots, p_{n}\right)\right) .
$$

As noted in Section II-D, this vector field is discontinuous, and its solution must be understood in the Filippov sense. Eq. (10) and Proposition 4.2 provide an expression of $\partial \mathcal{H}_{\mathrm{DC}}(P)$. One needs to first compute the generalized gradient, then compute the least-norm element, and finally project to each of the $n$ components. Note that the least-norm element of convex sets can be computed efficiently, see [16], however closed-form expressions are not available in general. One can also see that the compact set $Q^{n}$ is strongly invariant for $-\operatorname{Ln}\left(\partial \mathcal{H}_{\mathrm{DC}}\right)$ (cf. [10]).

Proposition 5.1: For the dynamical system (12), the generators' location $P=\left(p_{1}, \ldots, p_{n}\right)$ converges asymptotically to the set of critical points of $\mathcal{H}_{\mathrm{DC}}$.

Remark 5.2: The gradient dynamical systems enjoy convergence guarantees, but their implementation is centralized because of two reasons. First, all functions $G_{i}(P)$ need to be compared in order to determine which generator is active. Second, the least-norm element of the generalized gradients depends on the relative position of the active generators with respect to each other and to the environment. Moreover, as illustrated in Fig. 5, the evolution of the gradient system may not leave fixed even the generators that are circumcenters.

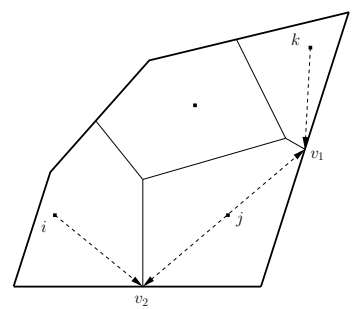

Fig. 5. Illustration of the gradient descent. The $j$ th generator is in the circumcenter of its own Voronoi region, but the control law (12) drives it toward the vertex $v$.

\section{B. Nonsmooth dynamical systems based on distributed gradients}

In this section, we propose a distributed implementation of the previous gradient dynamical system and explore its relation with behavior-based rules in multiple-vehicle coordination. Consider

$$
\dot{p}_{i}=-\operatorname{Ln}\left(\partial \lg _{V_{i}(P)}\right)(P),
$$


for $i \in\{1, \ldots, n\}$. Note that the system (13) is Voronoi-distributed, since $\operatorname{Ln}\left(\partial \lg _{V_{i}(P)}\right)(P)$ is determined only by $p_{i}$ and the position of its Voronoi neighbors $\mathcal{N}(P, i)$. For future reference, let $\operatorname{Ln}\left(\partial \lg _{\mathcal{V}}\right)(P)=$ $\left(\operatorname{Ln}\left(\partial \lg _{V_{1}(P)}\right)(P), \ldots, \operatorname{Ln}\left(\partial \lg _{V_{n}(P)}\right)(P)\right)$, and put $\dot{P}=$ $-\operatorname{Ln}\left(\partial \lg _{\mathcal{V}}\right)(P)$. Note also that $\operatorname{Ln}\left(\partial \lg _{\mathcal{V}}\right)(P)$ is discontinuous, and therefore its solutions must be understood in the Filippov sense. One can see that the compact set $Q^{n}$ is strongly invariant using the expression for the generalized gradient of $\lg$ in Proposition 3.1.

Proposition 5.3: Let $P \in Q^{n}$. Then the solutions of the dynamical system (13) starting at $P$ is unique.

Remark 5.4: (Relation with behavior-based robotics: move toward the furthest-away vertex) The distributed gradient control law (13) has an interesting interpretation in the context of behavior-based robotics. Consider the $i$ th generator. If the maximum of $\lg _{V_{i}(P)}$ is attained at a single vertex $v$ of $V_{i}$, then $\lg _{V_{i}(P)}$ is differentiable at that configuration, and its derivative corresponds to $\operatorname{versus}\left(p_{i}-v\right)$. Therefore, the law (13) corresponds to the behavior "move toward the furthest vertex in own Voronoi cell." If there are two or more vertices of $V_{i}$ where the value $\lg _{V_{i}(P)}\left(p_{i}\right)$ is attained, then (13) provides an average behavior by computing the least-norm element in the convex hull of all versus $\left(p_{i}-v\right)$ such that $\left\|p_{i}-v\right\|=\lg _{V_{i}(P)}\left(p_{i}\right)$.

Proposition 5.5: For the dynamical system (13), the generators' location $P$ converges asymptotically to the largest weakly invariant set contained in the closure of $A_{\mathrm{DC}}(Q)=$ $\left\{P \in Q^{n} \mid i \in I(P) \Longrightarrow p_{i}=\mathrm{CC}\left(V_{i}\right)\right\}$.

\section{Distributed dynamical systems based on geometric cen- tering}

Here, we propose an alternative distributed dynamical system for the multi-center function. Our design is directly inspired by the result in Theorem 4.3 on the critical points of the multi-center function $\mathcal{H}_{\mathrm{DC}}$. For $i \in\{1, \ldots, n\}$, consider the dynamical system

$$
\dot{p}_{i}=\mathrm{CC}\left(V_{i}\right)-p_{i}
$$

Alternatively, we may write $\dot{P}=\mathrm{CC}(\mathcal{V}(P))-P$. Note that this system is Voronoi-distributed and that the vector field (14) is continuous, since the circumcenter of a polygon depends continuously on the location of its vertices, and the location of the vertices of the Voronoi partition depends continuously on the location of the generators; see [14]. Having established the existence of solutions, one can also see that the compact set $Q^{n}$ is strongly invariant for the vector field $\mathrm{CC}(\mathcal{V})-\mathrm{Id}$. Next, we characterize the asymptotic convergence.

Proposition 5.6: For the dynamical system (14), the generators' location $P=\left(p_{1}, \ldots, p_{n}\right)$ converges asymptotically to the largest weakly invariant set contained in the closure of $A_{\mathrm{DC}}(Q)$.

\section{Simulations}

To illustrate the performance of the distributed coordination algorithms, we include some simulation results. The algorithms are implemented in Mathematica as a single centralized program. Measuring displacements in meters, we consider the domain determined by the vertices

$$
\begin{aligned}
\{(0,0),(2.5,0),(3.45,1.5) & (3.5,1.6),(3.45,1.7), \\
& (2.7,2.1),(1 ., 2.4),(.2,1.2)\} .
\end{aligned}
$$

In Figs. 6 and 7, we illustrate the performance of the dynamical systems (13) and (14), resp., minimizing the multi-circumcenter function $\mathcal{H}_{\mathrm{DC}}$. Observing the final configurations in the two figures, one can verify, visually and numerically, that the active generators are asymptotically centered as forecast by our analysis.
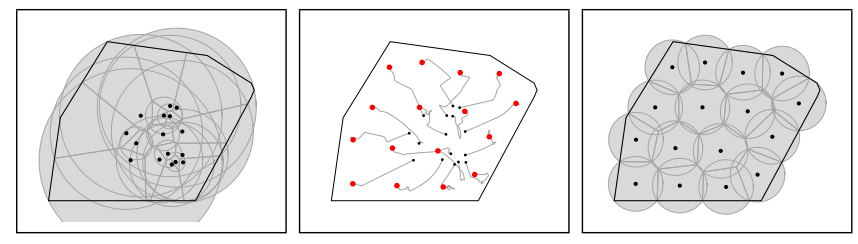

Fig. 6. "Toward the furthest" algorithm for 16 generators in a convex polygonal environment. The left (resp., right) figure illustrates the initial (resp., final) locations and Voronoi partition. The central figure illustrates the network evolution. After $2 \mathrm{~s}$, the value of $\mathcal{H}_{\mathrm{DC}}$ is approximately $.39504 \mathrm{~m}$.
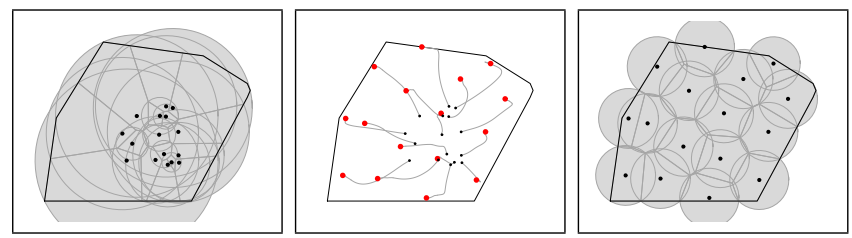

Fig. 7. "Move-toward-the-circumcenter" algorithm for 16 generators in a convex polygonal environment. The left (resp., right) figure illustrates the initial (resp., final) locations and Voronoi partition. The central figure illustrates the network evolution. After $20 \mathrm{~s}$, the value of $\mathcal{H}_{\mathrm{DC}}$ is approximately $0.43273 \mathrm{~m}$.

\section{CONCLUSIONS}

We have introduced a multi-center function that provide quality-of-service measures for mobile networks. We have analyzed its nonsmooth properties and characterized, under certain technical conditions, its critical points as center Voronoi configurations and as solutions of disk-covering problems. We have also considered various (distributed and non-distributed) algorithms that extremize the multi-center function. We have unveiled the remarkable geometric interpretations of these algorithms, discussed their distributed character and analyzed their asymptotic behavior using nonsmooth stability analysis. For futher details we refer the reader to the journal version of this work [10]. Future directions of research include: (i) sharpening the asymptotic convergence results for the proposed dynamical systems, (ii) considering 
the setting of convex polytopes in $\mathbb{R}^{N}, N>2$, and (iii) analyzing other meaningful geometric optimization problems and their relations with cooperative behaviors.

\section{Acknowledgments}

This work was supported by DARPA/AFOSR MURI Award F49620-02-1-0325 and ONR YIP Award N00014-03$1-0512$.

\section{REFERENCES}

[1] F. H. Clarke, Optimization and Nonsmooth Analysis, ser. Canadian Mathematical Society Series of Monographs and Advanced Texts. John Wiley, 1983.

[2] R. W. Brockett, "Dynamical systems that sort lists, diagonalize matrices, and solve linear programming problems," Linear Algebra and its Applications, vol. 146, pp. 79-91, 1991.

[3] U. Helmke and J. Moore, Optimization and Dynamical Systems. New York: Springer Verlag, 1994.

[4] A. F. Filippov, Differential Equations with Discontinuous Righthand Sides, ser. Mathematics and Its Applications. Dordrecht, The Netherlands: Kluwer Academic Publishers, 1988, vol. 18.

[5] A. Bacciotti and F. Ceragioli, "Stability and stabilization of discontinuous systems and nonsmooth Lyapunov functions," ESAIM. Control, Optimisation \& Calculus of Variations, vol. 4, pp. 361-376, 1999.

[6] D. Shevitz and B. Paden, "Lyapunov stability theory of nonsmooth systems," IEEE Transactions on Automatic Control, vol. 39, no. 9, pp. 1910-1914, 1994.

[7] S. P. Lloyd, "Least squares quantization in PCM," IEEE Transactions on Information Theory, vol. 28, no. 2, pp. 129-137, 1982, presented as Bell Laboratory Technical
Memorandum at a 1957 Institute for Mathematical Statistics meeting.

[8] R. M. Gray and D. L. Neuhoff, "Quantization," IEEE Transactions on Information Theory, vol. 44, no. 6, pp. 2325-2383, 1998, Commemorative Issue 1948-1998.

[9] Q. Du, V. Faber, and M. Gunzburger, "Centroidal Voronoi tessellations: Applications and algorithms," SIAM Review, vol. 41, no. 4, pp. 637-676, 1999.

[10] J. Cortés and F. Bullo, "Coordination and geometric optimization via distributed dynamical systems," SIAM Journal on Control and Optimization, vol. 44, no. 5, pp. 1543-1574, 2005.

[11] A. Suzuki and Z. Drezner, "The $p$-center location problem in an area," Location Science, vol. 4, no. 1/2, pp. 69-82, 1996.

[12] Z. Drezner, Ed., Facility Location: A Survey of Applications and Methods, ser. Springer Series in Operations Research. New York: Springer Verlag, 1995.

[13] M. de Berg, M. van Kreveld, M. Overmars, and O. Schwarzkopf, Computational Geometry: Algorithms and Applications, 2nd ed. New York: Springer Verlag, 2000.

[14] A. Okabe, B. Boots, K. Sugihara, and S. N. Chiu, Spatial Tessellations: Concepts and Applications of Voronoi Diagrams, 2nd ed., ser. Wiley Series in Probability and Statistics. New York: John Wiley, 2000.

[15] J. Cortés, S. Martínez, T. Karatas, and F. Bullo, "Coverage control for mobile sensing networks," IEEE Transactions on Robotics and Automation, vol. 20, no. 2, pp. 243-255, 2004.

[16] S. Boyd and L. Vandenberghe, "Convex optimization," Dec. 2002, preprint. 